\title{
Optical and electrochemical behaviour of the adsorption of potassium ethyl xanthate on gold
}

\author{
R.O. Lezna, N.R. de Tacconi and A.J. Arvia \\ Instituto de Investigaciones Fisicoquimicas Teóricas y Aphcadas (INIFTA), Facultad de Ciencias Exactas, \\ Unversidad Nacional de La Plata, Sucursal 4, Casilla de Correo 16, (1900) La Plata (Argentina)
}

(Received 16 November 1987; in revised form 27 April 1988)

\begin{abstract}
The first stages of the electroadsorption of ethyl xanthate (EX) on polycrystalline gold have been studied by optical and electrochemical techniques covering the $5 \times 10^{-6}$ to $1 \times 10^{-3} \mathrm{M}$ concentration range. According to ac voltammetry and electroreflectance measurements, the adsorption of EX begins at $-1.4 \mathrm{~V}$ (vs. SCE), in the HER potential region, followed by a capacitative process at $-1.0 \mathrm{~V}$. Both stages can be ascribed to the formation of different bonds between the EX and the gold surface through the sulphur atoms of the molecule. The following electroadsorption stage at $-0.7 \mathrm{~V}$ implies the formation of a surface compound leading subsequently to layer growth. Differential reflectivity was particularly useful in providing information on this type of complex adsorption process without appreciable interference from charging currents or stray faradaic reactions.
\end{abstract}

\section{INTRODUCTION}

Organic monolayers of well-defined structures supported on electrodes have proved useful for a variety of applications in microelectronics, batteries, biology, etc. Moreover, the fundamental problem of charge transfer across long distances is of considerable importance to electrochemical systems. This type of organic layer with variable although controlled distances provides a means of separating redox centres from metal surfaces in the process of electron tunnelling. However, progress has been restricted by the lack of precise characterization of these films and their defects [1].

From the practical point of view, the specific adsorption of collectors such as potassium ethyl xanthate (KEX) on mineral surfaces is used in the industrial process of flotation for separating different minerals from each other and from ore (gangue). The method has been applied to metallic sulphides for many years, although the fundamental chemistry of the adsorption-desorption processes is not properly understood [2-16]. In order to achieve a better understanding of the surface 
chemistry of xanthates, it is interesting to investigate the electrochemical behaviour of these substances on noble metals. In this respect gold is attractive because of its rather broad potential range where only double-layer processes can be observed. This region is therefore suitable for studying the adsorption of sulphur-containing compounds such as xanthates.

The electrochemistry of KEX on gold involves a series of complex reactions which take place in well-defined potential regions [2]. However, this work is concerned only with the early stages of the electroadsorption of KEX, leaving aside for the time being the dimerization of KEX to dixanthogen and other subsequent anodic reactions.

The adsorption of KEX on gold commences at rather negative potentials, partially overlapping the HER, and, given the characteristics of the processes involved, with a large capacitative component that tends to obscure the dynamic behaviour of the system. In situ UV-visible electroreflectance spectroscopy [17] appears to be a particularly useful tool to circumvent the interference of capacitative currents or stray faradaic processes, providing information additional to, and of a different kind from that obtained through electrochemical techniques.

\section{FXPERIMENTAL}

Electrochemical and optical measurements were made at $25^{\circ} \mathrm{C}$ using Kel-F encased discs of $\mathrm{Au}$ (99.99\%) of $7 \mathrm{~mm}$ diameter. The electrode was polished mechanically to a mirror finish with alumina of successively decreasing grades and freed of remaining particles in an ultrasonic bath before being introduced into the cell. The supporting electrolyte was $1 \mathrm{M} \mathrm{Na}_{2} \mathrm{SO}_{4}$ (Merck p.a.) and $\mathrm{KEX}$ was obtained from Fluka (puriss., p.a.). Solutions were prepared with Milli $\mathrm{Q}$ water that had been deoxygenated previously with purified nitrogen covering the $5 \times 10^{-6}$ to $10^{-3} M$ KEX concentration range. The electrolyte was degassed in the same manner prior to the introduction of KEX. All potentials were measured and are quoted against a SCE.

Electrochemical measurements were carried out with a model 173/179/175 PAR potentiostat-waveform generator system. Voltammograms were recorded, depending on the sweep rate, with a Houston $2000 \mathrm{X}-\mathrm{Y}$ recorder or with a Nicolet 2090 digital scope equipped with a model 206 plug-in and disk driver.

Ac voltammetric measurements were conducted by superimposing a small sine wave of less than $10 \mathrm{mV} \mathrm{p}-\mathrm{p}$ amplitude on a slow linear sweep rate of ca. $5 \mathrm{mV} / \mathrm{s}$. The corresponding ac response was demodulated with the aid of a two-phase lock-in amplifier and plotted in the form of either amplitude or in-phase signal.

Optical measurements were made with a specially designed spectrometer assembled in the laboratory for in situ studies of electrochemical systems. The set-up consists of a $250 \mathrm{~W}$ halogen lamp powered by a highly stabilized dc power supply coupled to a double monochromator to minimize stray light. The light is linearly polarized either parallel or perpendicular to the plane of incidence with the aid of a high transmission Glan Taylor polarizer and brought into the cell through a quartz 
window at an angle of incidence of $56^{\circ}$. All the lenses and cell optical windows are made of quartz. Diffractions orders higher than one are suppressed by suitable filters.

The radiation reflected off the surface of the electrode is focused onto a photomultiplier with sensitivity S20 energized by a programmable power supply Kepco OPS 2000B. A feedback system was designed to control the output of the power supply in order to keep the anode current of the tube constant. This procedure allows the slow changes in reflected intensity to be normalized against variations arising from the lamp spectral curve, the optical transfer function of the system, etc. The small fast changes in reflected intensity, typically 1 part in 10000 or lower, brought about by modulating the electrode potential by either a small sine wave or square wave, are rectified by a two-phase lock-in amplifier, NF model L1575, in those cases where time-dependent information is not relevant to the aim of the measurement. On the other hand, when the time dependence of the optical transients arising, for instance, from potentiostatic pulses contains the required information, the output of the photomultiplier is amplified by a low-noise ac amplifier, and then the signal is averaged on a model 4203 PAR signal averager. Most of the machines are on the IEEE-488 interface bus with a Hewlett-Packard personal computer as the controller. Experiment automation and data gathering/ processing were conducted with this set-up.

RESULTS

\section{Conventional voltammetric data}

Cyclic voltammetric experiments were conducted in order to get a rough picture of the system behaviour and to delimit the potential regions of the different processes.

Voltammograms run at $70 \mathrm{mV} / \mathrm{s}$ for $1 \times 10^{-4} M$ KEX (Fig. 1a) and spanning the potential region positive of the HER, between -1.2 and $0.65 \mathrm{~V}$, show two anodic current peaks, one at $-0.07 \mathrm{~V}$ (peak Ia) and another one at $0.04 \mathrm{~V}$ (peak IIa). Peak Ia, as will be shown later in this work, is related to the first stages of the electroadsorption of EX, whereas peak IIa corresponds to the dimerization of EX to dixantogen $(\mathrm{EX})_{2}$ [2]. Either two or three peaks are observed on the negative scan depending on the upper reversing potential and corresponding to different steps in the electroreduction of $(\mathrm{EX})_{2}$ (peaks IIc and $\mathrm{II}_{\mathrm{c}}^{\prime}$ ) and to the electrodesorption of EX (peak Ic).

The voltammogram changes considerably when the concentration of KEX is increased to $10^{-3} M$ (Fig. 1b). Thus, an abrupt increase in the anodic current can be observed in the potential range of peak IIa. On the other hand, the negative scan continues to exhibit peaks Ic, II'c and IIc. The dimerization of EX to (EX) $)_{2}$ at potentials greater than $-0.2 \mathrm{~V}$ is enhanced considerably in $10^{-3} \mathrm{M}$ KEX.

These voltammetric data are useful for choosing the most suitable experimental conditions, namely potential limits, concentration range, sweep rate, etc., to investigate the adsorption of EX on gold. 


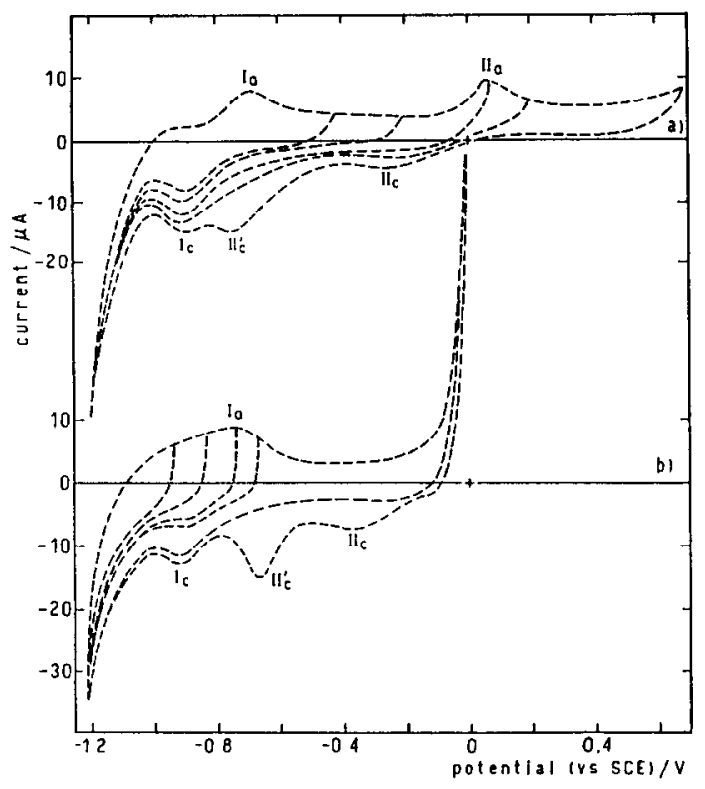

Fig. 1. Voltammograms run at $70 \mathrm{mV} / \mathrm{s}$ with different upper limits for (a) $[\mathrm{KEX}]=1 \times 10^{-4} \mathrm{M}$ and (b) [KEX] $=1 \times 10^{-3} M$ in $0.1 M \mathrm{Na}_{2} \mathrm{SO}_{4}$.

The voltammograms run at $50-70 \mathrm{mV} / \mathrm{s}$ between -1.2 and $-0.3 \mathrm{~V}$ in KEXcontaining solutions are appreciably different from the blank run (Fig. 2), even at the lowest concentration of KEX, i.e. $5 \times 10^{-6} M$. The blank run exhibits a rather large potential range associated with electrical double-layer (edl) charging and the contribution of the HER from ca. $-0.9 \mathrm{~V}$ downwards.

The voltammograms resulting from $5 \times 10^{-6} M$ KEX (Fig. 2a) are made up, to a large extent, of contributions from double-layer charging with a small hump on the positive scan at ca. $-0.95 \mathrm{~V}$ (peak I'a) and a current peak on the negative sweep at ca. $-1.0 \mathrm{~V}$ (peak I'c) largely overlapping the HER, this reaction being shifted to more positive potentials than in the blank run on account of the presence of sulphur-containing species on the electrode.

For $1 \times 10^{-4} M$ KEX (Fig. 2b), peak Ia is recorded at $50 \mathrm{mV} / \mathrm{s}$ as a broad maximum at $-0.7 \mathrm{~V}$ in addition to the small maximum at $-0.95 \mathrm{~V}$ (peak $\mathrm{I}^{\prime} \mathrm{a}$ ). It should be noted that the negative scan displays a profile which is to some extent more complex than its positive counterpart. However, the two cathodic peaks at -0.7 and $-0.82 \mathrm{~V}$ were found to merge into a single peak (Ic) as the scan rate was slowed down to $10 \mathrm{mV} / \mathrm{s}$, although this result is not shown in Fig. 2 .

At a higher KEX concentration, $1 \times 10^{-3} \mathrm{M}$, peak Ia can also be recorded at $-0.7 \mathrm{~V}$ but now the complementary cathodic maximum (Ic) is also clearly defined (Fig. 2c). Peak Ia was not detected at concentrations lower than $5 \times 10^{-5} M$ KEX and its corresponding reduction, Ic, depends on both the concentration of KEX in 

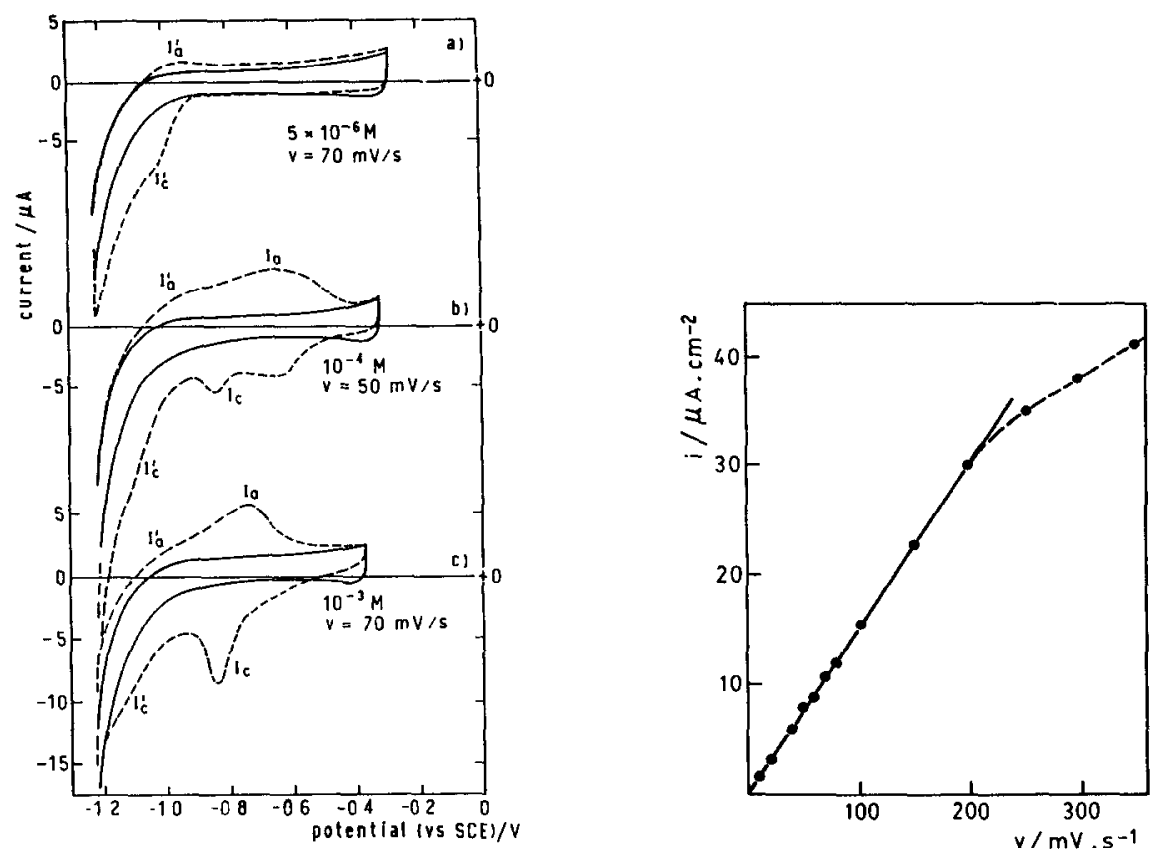

Fig. 2. Voltammograms run for different KEX concentrations. Upper limit $=-1.2 \mathrm{~V}$, lower limit $=<$ -0.3 V. (a) $[\mathrm{KEX}]=5 \times 10^{-6} M$; (b) $[\mathrm{KEX}]=1 \times 10^{-4} M$; (c) $[\mathrm{KEX}]=1 \times 10^{-3} M$. (—) Blank run.

Fig. 3. $i / v$ plot corresponding to peak Ia. $[\mathrm{KEX}]=1 \times 10^{-3} \mathrm{M}$.

solution and on the time the current after peak Ia is allowed to tail into more positive potentials.

The height of peak Ia varies linearly with the scan rate, $v$, up to $200 \mathrm{mV} / \mathrm{s}$ although this relationship no longer holds at higher sweep rates (Fig. 3). The voltammetric charge estimated for peak Ia is about $70 \mu \mathrm{C} / \mathrm{cm}^{2}$, a charge density value which exceeds $55 \mu \mathrm{C} / \mathrm{cm}^{2}$. The latter corresponds to the monolayer charge for EX, obtained on the basis that the projected area is $0.29 \mathrm{~nm}^{2}[18]$ and that the electroadsorption process involves one electron per molecule of EX.

Therefore, cyclic voltammetry offers an outline of the complex processes involved in the adsorption of KEX on gold. This picture is, however, still rather incomplete and to some extent obscured by the partial overlap of the EX adsorption with the HER, which opens up the possibility that other stages of the EX adsorption at potentials more negative than $-1.2 \mathrm{~V}$ are left undetected. Should there be such a process, coverages measured by this technique from $-1.2 \mathrm{~V}$ towards more positive potentials must be added to the amount of EX adsorbed, if any, at potentials negative of $-1.2 \mathrm{~V}$ within the HER region. 


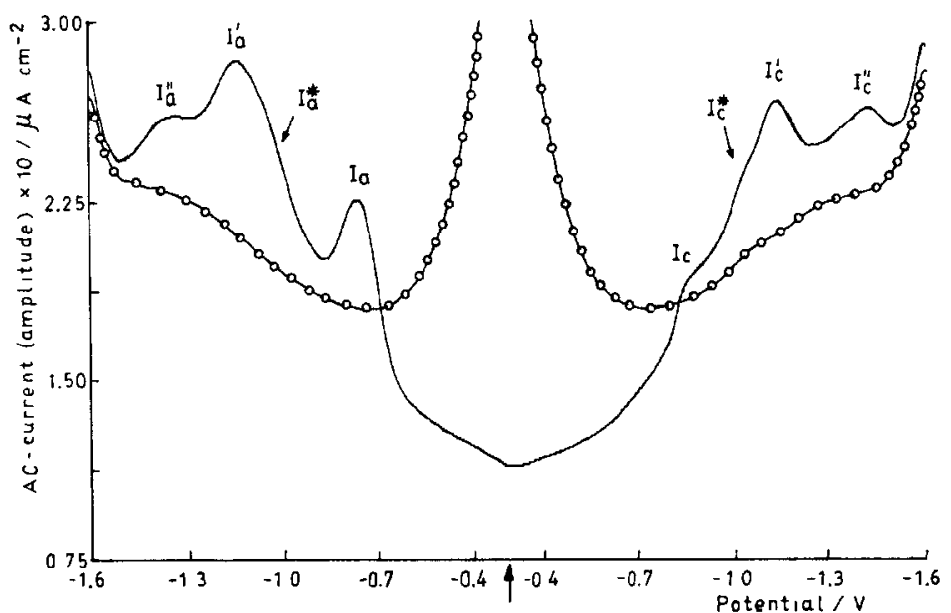

Fig. 4. Ac voltammograms for the $\mathrm{Au}$ electrode with $\left.0.1 M \mathrm{Na}_{2} \mathrm{SO}_{4}\right)(\mathrm{O}-\mathrm{O})$ and with $0.1 M$ $\mathrm{Na}_{2} \mathrm{SO}_{4}+10^{-3} \mathrm{M} \mathrm{KEX}$ ( $\longrightarrow$ ). The potential was scanned cyclically from $-1.6 \mathrm{~V}$ up to $-0.3 \mathrm{~V}$. Ac potential amplitude $\Delta E=10 \mathrm{mV}$ p-p; frequency $f=100 \mathrm{~Hz}$.

Ac voltammetry and in situ reflectance measurements under comparable experimental conditions

In order to improve on the resolution of the different stages of the adsorption of EX on gold, we tried in situ reflectance measurements and ac voltammetry. The latter, although it is also affected by the presence of large charging currents, yielded better results than cyclic voltammetry. Differential reflectivity proved to be the most useful in overcoming these difficulties, providing, in addition, spectral information. Optical measurements were conducted in the spectral region between 3.5 and $1.4 \mathrm{eV}$. Potassium ethyl xanthate in solution is known to be transparent in this range of energies [7].

To check whether small amounts of sulphur-containing species were on the surface at high negative potentials, in the HER region, as has been reported for the case of sulphur on silver [19], we looked for these species within a few hundred $\mathrm{mV}$ in this region.

Figure 4 shows the ac voltammograms (signal amplitude vs. potential) corresponding to $1 \times 10^{-3} M \mathrm{KEX}$ and to the supporting electrolyte. The potential was scanned cyclically from $-1.6 \mathrm{~V}$ up to $-0.3 \mathrm{~V}$ and modulated at $100 \mathrm{~Hz}$ (frequency of better resolution).

The ac voltammogram of the KEX-containing solutions shows, in addition to a relatively large capacitative component, the occurrence of three processes in the positive scan characterized by peaks I" $a, I^{\prime}$ a and Ia, and also a very small hump, $I^{*} a$, which is very difficult to analyse at this stage of the presentation. Correspondingly, the negative scan shows a complementary response (peaks Ic, $I^{\prime \prime} c, I^{\prime} c$ and $I^{*} c$ ) although this is not exactly symmetric. 


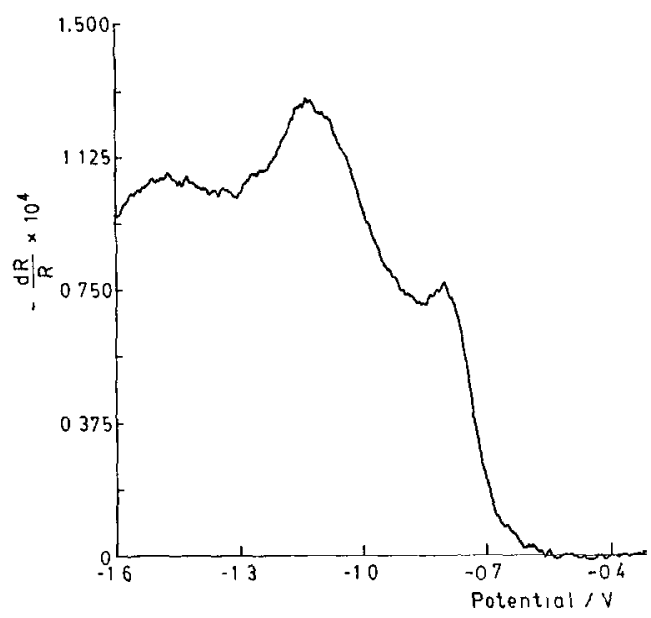

Fig. 5. In-phase differential reflectivity vs. potential curve, positive scan. Initial potential $=-1.6 \mathrm{~V}$. Angle of incidence $\phi=59^{\circ}$; photon energy $=2.5 \mathrm{eV}$; p-polarization; $\Delta E=50 \mathrm{mV} \mathrm{p}-\mathrm{p} ; f=220 \mathrm{~Hz}$. $[\mathrm{KEX}]=1 \times 10^{-3} M$.

On the other hand, Fig. 5 shows the differential reflectivity (in-phase component) $\mathrm{d} R / R$ at $2.5 \mathrm{eV}$ during a positive scan for the same concentration. The best results were obtained in this case at $220 \mathrm{~Hz}$ as this frequency helped in suppressing, to a certain extent, any distortion which may arise from the HER. At the potentials employed, there is no growth of hydrogen bubbles which would have introduced large spikes in the optical response. Similar optical measurements were conducted successfully for the adsorption of sulphide on gold which was shown also to overlap the onset of the HER [20].

A similar profile was obtained by both ac voltammetry and differential reflectivity; that is, three main processes are seen to occur on the positive scan ( $I^{\prime \prime} \mathrm{a}, \mathrm{I}^{\prime} \mathrm{a}$ and Ia). Thus, in addition to the processes giving rise to peaks $I^{\prime} \mathbf{a}$ and Ia, already detected by cyclic voltammetry (Fig. 2), another stage $I^{\prime \prime}$ a shows up at ca. $-1.4 \mathrm{~V}$, providing evidence for the existence of adsorbed species on the electrode at high negative potentials.

Furthermore, ac voltammetry also shows a net decrease in the capacitative contribution in the -0.6 to $-0.3 \mathrm{~V}$ range which is due to the complete coverage of the electrode by the electroadsorbate. According to cyclic voltammetry, at potentials more positive than $-0.6 \mathrm{~V}$ the monolayer charge expected for full coverage of the electrode by EX has already been exceeded. Correspondingly, peak Ia in the optical signal is also followed by a rapid decrease close to zero in the potential region where the monolayer charge is exceeded.

\section{Potentiostatic step experiments}

A programme of potentiostatic steps was applied to the gold electrode with a view to determining the equilibrium coverage of $\mathrm{EX}$ as a function of the potential. 

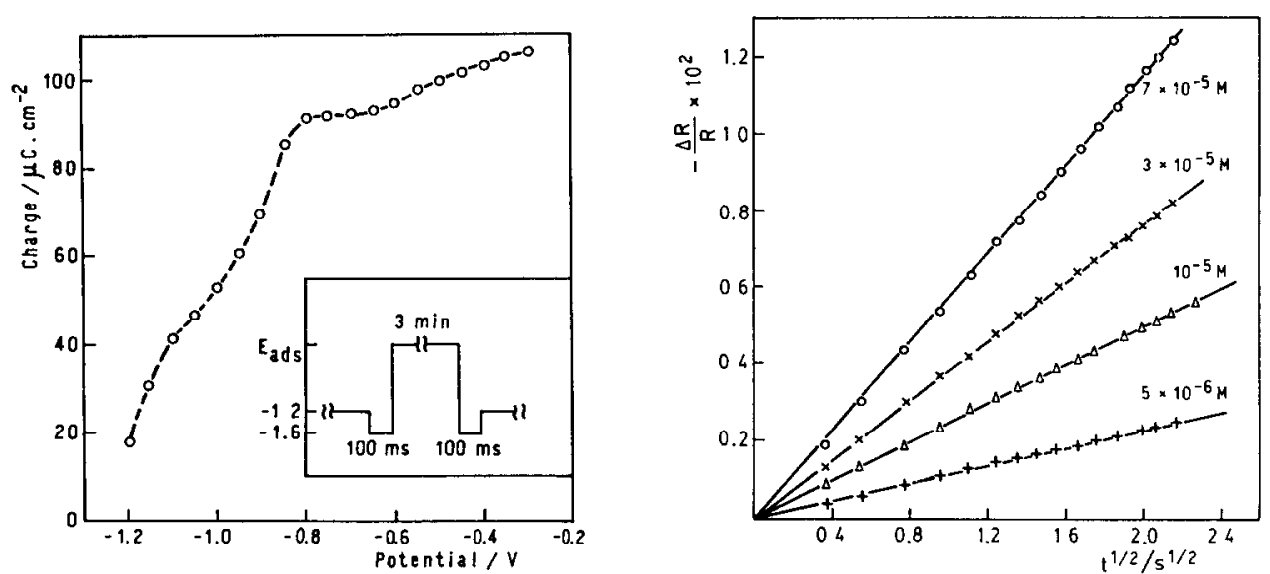

Fig. 6. Dependence of the amount of adsorbed EX on potential for $1 \times 10^{-3} M$ KEX. Inset: measuring potential programme.

Fig. 7. Time dependence of the reflectance change at $1.84 \mathrm{eV}$ following a potential step from -1.2 to $-0.32 \mathrm{~V}$ at various $\mathrm{KEX}$ concentrations in $0.1 \mathrm{M} \mathrm{Na}_{2} \mathrm{SO}_{4}$.

For this purpose, a $1 \times 10^{-3} M$ KEX solution + supporting electrolyte was employed, taking into account that according to the results shown in Fig. 4 complete desorption is achieved only at $-1.6 \mathrm{~V}$. Cathodic rather than anodic transients were integrated owing to the fact that the charge vs. time curves obtained from the anodic steps exhibited some distortion at long times (ca. $5 \mathrm{~s}$ ) because of the presence of a small cathodic standing current even at the most positive potentials used. This residual current cannot arise from traces of oxygen in the solution as careful precautions were taken to eliminate oxygen from the electrochemical cell. However, had this been the case, traces of oxygen would have been reduced by EX in the solution [13]. The residual current results probably from small hydrogen currents at gold [21]. Besides, the presence of KEX brings about a catalytic effect on the HER, as already described in the analysis of the voltammetric data.

The perturbing potential programme used in the potentiostatic steps is depicted in Fig. 6. It was selected so as to have, for a given potential, an uncovered gold surface both at the beginning and at the end of the measurement with minor interference from the HER. Thus, starting from $-1.2 \mathrm{~V}$, the electrode was cleaned of adsorbate by stepping the potential to $-1.6 \mathrm{~V}$ for $100 \mathrm{~ms}$, then to $E_{\text {ads }}$ for $3 \mathrm{~min}$ to reach equilibrium, and finally stepped back to $-1.6 \mathrm{~V}$ to record the current transient. After $100 \mathrm{~ms}$, the potential was set back to $-1.2 \mathrm{~V}$ to preclude the formation and growth of hydrogen bubbles. The current transient between $E_{\text {ads }}$ and $-1.6 \mathrm{~V}$ was integrated as a function of the time and the resulting curve was extrapolated to zero time to obtain the amount adsorbed at $E_{\text {ads }}$.

The charge density vs. potential plot (Fig. 6) from -1.2 to $-0.3 \mathrm{~V}$ obtained after baseline correction from a blank run reveals an initial charge density of ca. 18 
$\mu \mathrm{C} / \mathrm{cm}^{2}$ followed by two plateaux, with the second one being, in this case, much better defined than the first. Therefore, if the initial charge of ca. $18 \mu \mathrm{C} / \mathrm{cm}^{2}$ is assigned to peak I" a as detected by ac voltammetry, the two waves observed in the charge vs. potential curve should correspond to peaks I'a and Ia, respectively.

It should be noted that the coverage from $-1.6 \mathrm{~V}$ to ca. $-1.0 \mathrm{~V}$, i.e. in the potential range of peaks I'a and I'a, is close to that expected for a monolayer of EX, whereas when the potential range is extended to include the region of peak Ia the coverage reaches a value of about twice that of a monolayer.

\section{Optical measurements}

Optical transients were obtained at $1.84 \mathrm{eV}$, away from the absorption edge of gold, by stepping the potential from -1.2 to $-0.32 \mathrm{~V}$. A fast change in reflectivity was observed during the first $10 \mathrm{~ms}$ arising from the electroreflectance effect of gold. This initial change was subtracted from the optical transients to obtain the linear $\Delta R / R$ vs. $t^{1 / 2}$ plots (Fig. 7) for concentrations ranging from $5 \times 10^{6}$ to $7 \times 10^{-5} M$ KEX. The results obtained indicate the proportionality between the amount of adsorbate on the surface and the change in the reflectance of the electrode; that is, the optical effects of charge and coverage should be independent of one another.

For KEX concentrations below $1 \times 10^{-5} M$, the slope of the $\Delta R / R$ vs. $t^{1 / 2}$ plots were found to be proportional to the corresponding KEX concentrations, indicating pure diffusion control for the electroadsorption of EX. At KEX concentrations greater than $1 \times 10^{-5} M$, the slopes of the straight lines are smaller than those

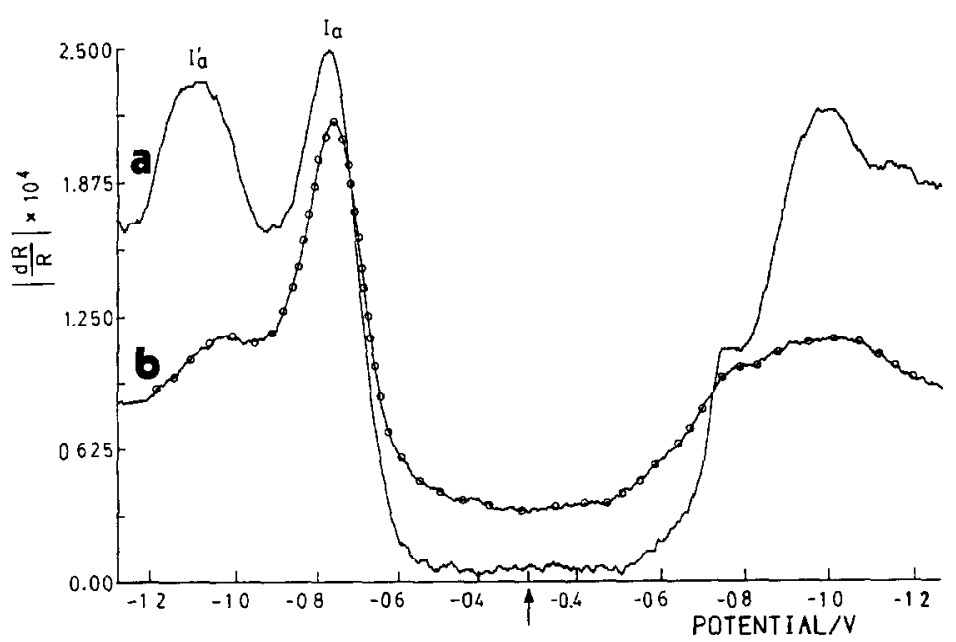

Fig. 8. In-phase differential reflectivity potential curves during a positive and a negative scan. $\phi=59^{\circ}$; p-polarization; $\Delta E=50 \mathrm{mV}$ p-p; $f=22 \mathrm{~Hz}$. (a) $2.5 \mathrm{eV}$; (b) $1.84 \mathrm{eV}$. 
expected for pure diffusion kinetics. Thus, the overall process seems to be under mixed control.

Differential reflectivity vs. potential curves started at $-1.2 \mathrm{~V}$, i.e. outside the HER region, are displayed in Fig. 8 for $1 \times 10^{-3} M$ KEX. Two main processes can be detected on the positive scan followed by a rapid decrease in the reflectivity for potentials greater than $-0.7 \mathrm{~V}$. The main change in the response when the initial potential is now $-1.2 \mathrm{~V}$ lies with peak Ia, which is much sharper than I'a, and more clearly resolved in this experiment. As for the spectral dependence, peak I'a follows
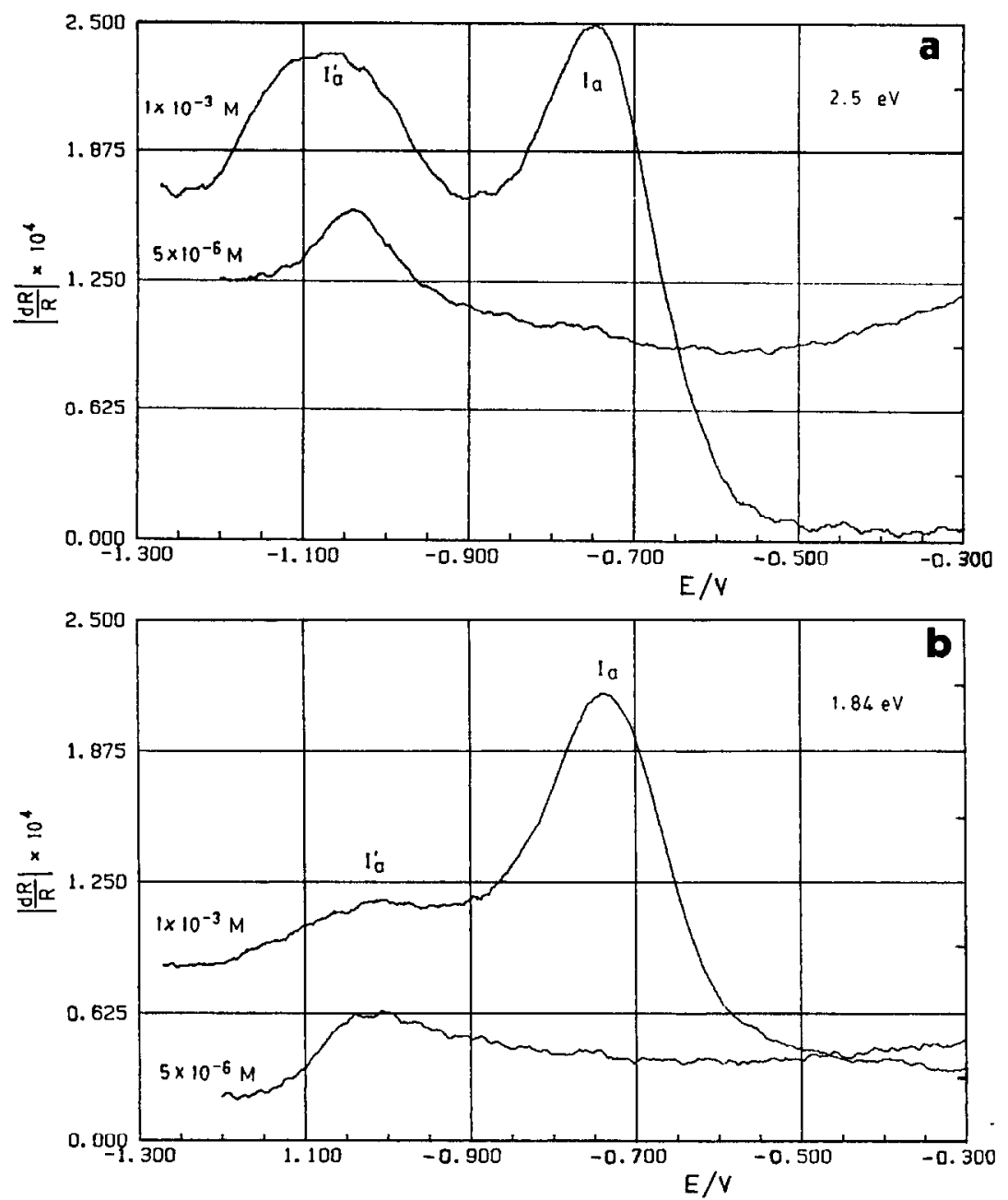

Fig. 9. Energy dependence of the differential reflectivity curves for $5 \times 10^{-6} M$ and $1 \times 10^{-3} M$ KEX. (a) $2.5 \mathrm{eV}$; (b) $1.84 \mathrm{eV}$. 
the intensity of the spectrum for bare gold; that is, it appears attenuated at $1.84 \mathrm{eV}$ as compared with the curve at $2.5 \mathrm{eV}$. On the other hand, the maximum at $-0.7 \mathrm{~V}$ (Ia), reveals no strong dependence on the wavelength as its intensity undergoes just a small change in going from 2.5 to $1.84 \mathrm{eV}$. This behaviour of peak Ia is characteristic of species strongly bound to the metal which alters markedly the spectrum of bare gold. On the negative scan, two processes related to the electroadsorption of EX can be observed although with a poorer resolution than their corresponding anodic pairs. Hence, the analysis of differential reflectivity will be mainly restricted to positive scans.

In order to determine the influence of the KEX concentration on peaks $I^{\prime} a$ and Ia, the differential reflectivity for $1 \times 10^{-3} M$ and $5 \times 10^{-6} M$ KEX solutions was measured for photon energies of 2.5 and $1.84 \mathrm{eV}$ (Fig. 9). The species giving rise to peak I'a shows up at all KEX concentrations, although the potential of peak I'a is shifted towards more negative values as the concentration of KEX is increased. Conversely, peak Ia is not detected at the lowest concentration of KEX, i.e. $5 \times 10^{-6} M$. This result is in keeping with the data obtained by cyclic voltammetry described previously (Fig. 2a).

From the results of Fig. 9 we can conclude that the adsorption of EX on gold occurs first in the potential range of peak $I^{\prime}$ a and when the EX surface concentration exceeds a certain threshold value corresponding to ca. $1 \times 10^{-4} M$ in the bulk, the electroadsorption stage at $0.7 \mathrm{~V}$ brings about the appearance of the relatively sharp peak Ia. Thereafter a sharp fall in the differential electrode reflectivity is observed due to the growth of multilayers. The height of peak Ia does not change markedly with the photon energy as does peak I'a.

The effect of the modulation frequency on the differential reflectivity vs. potential curves at $2.5 \mathrm{eV}$ for $1 \times 10^{-3} M$ KEX involving peaks $\mathrm{I}^{\prime} \mathrm{a}$ and $\mathrm{Ia}$ is rather

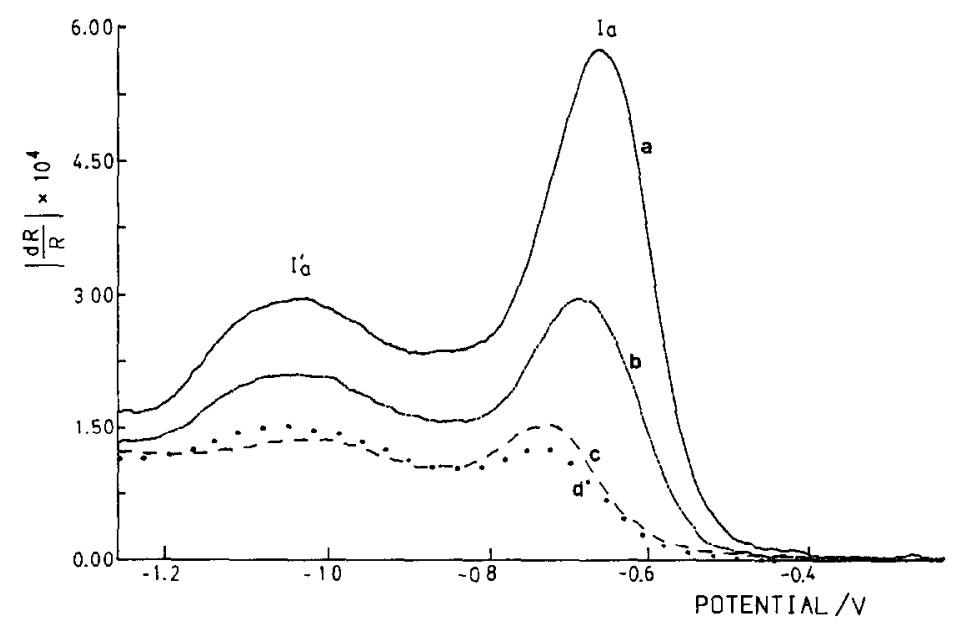

Fig. 10. Differential reflectivity vs. potential curves for $1 \times 10^{-3} M$ KEX. Initial potential: $-1.27 \mathrm{~V}$; $\phi=59^{\circ} ; 50 \mathrm{mV} \mathrm{p}-\mathrm{p} ; \lambda=2.5 \mathrm{eV} . f=$ (a) $20 \mathrm{~Hz}$; (b) $40 \mathrm{~Hz}$; (c) $80 \mathrm{~Hz}$; (d) $132 \mathrm{~Hz}$. 
distinctive for these two peaks (Fig. 10). Thus, peak I'a is broader than peak Ia and much less influenced by the frequency so that the peak Ia/peak I'a height ratio increases as the frequency decreases. On the other hand, both peak potentials shift towards more negative values as the frequency is varied from 20 to $180 \mathrm{~Hz}$.

\section{DISCUSSION}

As the first stage of the EX adsorption takes place in the HER region, at ca. $-1.4 \mathrm{~V}$, not enough information is available to draw conclusions concerning the nature of the adsorbate. However, as peak $\mathrm{I}^{\prime \prime} \mathrm{a}$ is well defined at comparatively high frequencies, i.e. $100 \mathrm{~Hz}$, it could be ascribed to a capacitative type of adsorption. This first adsorption stage could arise from the bonding of EX to the gold surface through its uncharged sulphur atom, allowing for the rather negative charge of the electrode in this potential region.

The second stage of EX adsorption, related to peak I'a, was found in the entire KEX concentration range covered in the present work. The kinetics of the adsorption process become diffusion-controlled for concentrations lower than $5 \times 10^{-5} M$, as seen in Fig. 7. The optical response corresponding to $I^{\prime}$ a becomes larger and the peak potential shifts towards negative values with increasing KEX concentration (Fig. 9) as is expected for an adsorption-desorption process [22]. Furthermore, the ac impedance in the potential range of peak $I^{\prime} a$, as determined through ac voltammetry (Fig. 4), is determined primarily by the quadraturc component. This fact provides evidence in favour of the capacitative nature of the second adsorption stage of EX on gold. In this case, the EX-gold interaction should involve the second (charged) sulphur atom of EX, probably in ionic form, leading the adsorbate into a more ordered structure in that the aliphatic tail should now be oriented towards the solution side, with the two sulphur atoms interacting directly with the gold surface. Besides, this configuration should hinder to some extent the librational modes of the adsorbate. The fact that peak I'a is rather broad can be interpreted in terms of mutual adsorbate interactions. This behaviour of the second adsorption stage is to be expected if the charged sulphur atoms are adsorbed without transferring their charge entirely. The spectral dependence of peak I'a follows that of bare gold, at least qualitatively (Fig. 8). Hence, the interactions of EX through the sulphur atoms with the gold surface should be sufficiently weak for the spectrum of gold not to be appreciably modified in shape.

The adsorption step measured at about $-0.7 \mathrm{~V}$, peak Ia, is observed only for concentrations greater than $5 \times 10^{-5} M$ KEX (Figs. 2 and 9). That is, for this process to take place a certain surface concentration of EX adsorbate is required. This requirement is known to be typical of compound formation [23]. The strong frequency dependence of this process indicates the slowness of this step as compared with those of the first and second adsorption stages already discussed (Fig. 10). Peak Ia should be related to a process of charge transfer, as was determined by cyclic voltammetry. The most likely process is the discharge of the ionic sulphur atom giving rise to the formation of a surface compound. Subsequently, the growth 


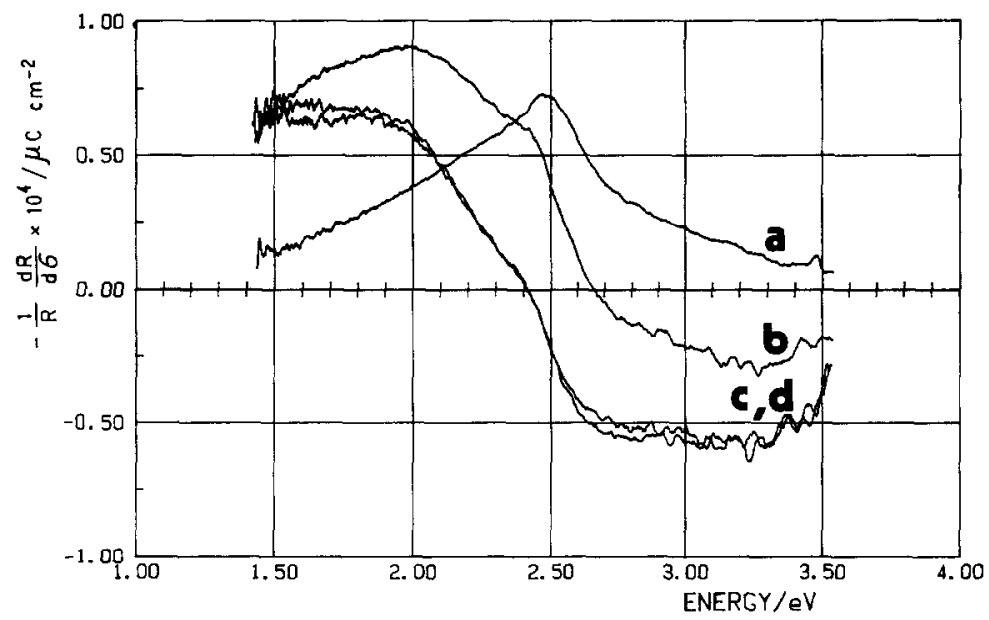

Fig. 11. Charge normalized electroreflectance spectra taken at different potentials. $\phi=59^{\circ} ; 60 \mathrm{mV} p-p$; $132 \mathrm{~Hz}$; p-polarization. (a) $-1.2 \mathrm{~V}$; (b) $-0.9 \mathrm{~V}$; (c) $-0.42 \mathrm{~V}$; (d) $-0.32 \mathrm{~V}$.

of this layer continues as the potential is made more positive. It is worthwhile mentioning that peaks I'a and Ia for EX on gold are somewhat similar to those observed for the adsorption of sulphide on gold [20].

The spectral variation of the intensity of peak Ia shows no correlation with that for the spectrum of bare gold as it is displayed with comparable intensity at $1.84 \mathrm{eV}$ and $2.5 \mathrm{eV}$ (Fig. 9). Therefore, the strong interaction between the adsorbed layer at $-0.7 \mathrm{~V}$ and the metal modifies the electroreflectance spectrum of the substrate significantly.

The optical response of the surface coverage of $\Lambda \mathrm{u}$ brought about by the electroadsorption of EX depends strongly on both the photon energy and the bias potential for a given concentration. The spectra shown in Fig. 11 were taken at four bias potentials chosen so as to reveal the distinctive contributions of the different stages in the process of adsorption. The response in current of the electrochemical cell to the potential modulation was integrated, for a given bias potential, and the resulting charge, $\sigma$, was used to normalize the corresponding spectrum.

The differential reflectivity measured at $-1.2 \mathrm{~V}$ (Fig. 11), although with the same sign as and a similar shape to those of EX-free gold [24], tails off much more slowly into the red region than the curve for the uncovered surface due to the amount of EX adsorbed at ca. $-1.4 \mathrm{~V}$ as mentioned earlier. The differential reflectivity is given by

$$
\frac{1}{R} \frac{\mathrm{d} R}{\mathrm{~d} E}=\frac{1}{R}\left[\left(\frac{\partial R}{\partial E}\right)_{\theta}+\sum_{i}\left(\frac{\partial R}{\partial \theta_{i}}\right)_{E} \frac{\mathrm{d} \theta_{i}}{\mathrm{~d} E}\right]
$$

where $\theta$ represents the surface concentration of the various types of EX species on the electrode. For uncovered gold, the second summation term within the brackets is 
zero, whereas for the EX-covered surfaces the second term should be predominant at low modulation frequencies. Curve a in Fig. 10 is determined mainly by the electroreflectance effect of gold, $(1 / R)(\partial R / \partial E)_{\theta}$, as the residual coverage at -1.2 $\mathrm{V}$ is rather small (Fig. 6).

The modifications to the spectra introduced by the processes I'a (ionic adsorption) and the beginning of the compound formation (foot of wave Ia in Fig. 8) at $-0.9 \mathrm{~V}$, is displayed by spectrum $\mathrm{b}$ in Fig. 11. The response is positive at high energies, then it undergoes a sign change near the absorption edge of gold $(2.5 \mathrm{eV})$, and shows a broad band at around $2 \mathrm{eV}$. This band cannot be ascribed to an increase in roughness [25] brought about by the adsorbate as the spectra of EX-free gold before and after the adsorption reveal no differences. The rather high frequency of the modulation, $132 \mathrm{~Hz}$, and the bias potential chosen (in between adsorption-desorption regions) are suitable conditions for the coefficient $(\partial R / \partial E)_{\theta}$ to make a significant contribution to the spectrum of curve $b$. That is, the band in the red region $(2 \mathrm{eV})$ is likely to arise from the electronic properties of gold modified by the formation of a covalent bond through the discharge of the sulphur anion. The coverage with the species giving rise to process Ia should be small as the bias potential is located on the negative side of the peak position. A similar band at around $2 \mathrm{eV}$ has been detected under similar experimental conditions on a gold electrode covered with a small surface concentration of strongly bound sulphide [20]. For a thicker layer, about two monolayers, at -0.42 and $-0.32 \mathrm{~V}$, a decrease in intensity and a loss of resolution are observed clearly (curves $c$ and d, Fig. 11). However, the trend observed in curve $b$ is further confirmed by what is measured at higher (or multilayer) coverages leading to an optical behaviour somewhat similar to that observed for the UPD of lead on Au [26]. In spite of the differences between the two types of adsorbates, the strong interaction with the substrate seems to be the common characteristic of both systems.

The surface layer thicknesses associated with curves $c$ and $d$ exceed that of a monolayer. Therefore, the coincidence of these curves can presumably be related to the fact that due to the insulating characteristics of the layer, the outermost part of it can see only an attenuated form of the potential modulation.

\section{CONCLUSION}

The first stages of the electroadsorption of EX on polycrystalline gold have been studied by using a proper combination of optical and electrochemical techniques. The adsorption was found to take place in three steps: two processes at $-1.4 \mathrm{~V}$ and at $-1.0 \mathrm{~V}$, which can be ascribed to the formation of bonds between the EX molecule and the gold surface through its two sulphur atoms, uncharged and charged respectively. A pseudo-faradaic mechanism at $-0.7 \mathrm{~V}$ accounts for the formation of a surface compound followed by the growth of multilayers.

The strong interaction between the electrode and the adsorbed KEX molecule arising from the formation of a surface compound is revealed by the modifications 
introduced to the electronic properties of gold, whose spectrum shows a new band at ca. $2 \mathrm{eV}$ in the corresponding potential region. A similar band has been detected for a gold electrode covered with strongly bound sulphide.

The use of in situ electroreflectance measurements and ac voltammetry have made possible the observation of the different stages in the adsorption of KEX on gold, even inside the HER region, without appreciable interference from capacitative currents or stray faradaic reactions.

\section{ACKNOWLEDGEMENTS}

This research project was supported financially by the Consejo Nacional de Investigaciones Científicas y Técnicas and the Comisión de Investigaciones Científicas de la Provincia de Buenos Aires. This work was also supported partially by the Regional Program for the Scientific and Technological Development of the Organization of the American States. Part of the equipment used in the present work was provided through the co-operation agreement between the University of Mainz (F.R.G.) and the University of La Plata (Argentina).

\section{REFERENCES}

1 Symposium on Critical Issues in the Structure and Chemistry of Organic Monolayers at Electrode Surfaces, 171st Meeting of the Electrochemical Society, Philadelphia, 10-15 May 1987.

2 O. Huynh Thi, M. Lamache and D. Bauer, Electrochim. Acta, 26 (1981) 33.

3 J. Mielczarski, F. Werfel and E. Suoninen, Appl. Surf. Sci., 17 (1983) 160.

4 D. Schuhmann, A.M. Guinard-Baticle and M. Lamache, Electrochim. Acta, 28 (1983) 79.

5 P.E. Richardson, S. Srinivasan and R. Woods, Proc. Int. Symp. on Electrochemistry in Mineral Processing, Vol. 84-10, The Electrochemical Society, Pennington, 1984.

6 S.V. Pihlajamaki and J.J. Kanbare, J. Electroanal. Chem., 170 (1984) 213.

7 O. Lam-Thi, M. Lamache and D. Bauer, Electrochim. Acta, 29 (1984) 217.

8 S. Kelebek and G.W. Smith, Int. J. Miner. Process., 14 (1985) 275.

9 I.C. Hamilton and R. Woods, Langmuir, 2 (1986) 770.

10 J. Mielczarski, Colloids Surf., 17 (1986) 251.

11 A. Ivaska and J. Leppinen, Talanta, 33 (1986) 801.

12 L.S. Johanson, J. Juhanova, K. Laajalehto, E. Seroninen and J. Mielczarski, Surf, Interfacial Anal., 9 (1986) 501.

13 J.O. Leppinen and J.K. Rastas, Colloids Surf., 20 (1986) 221.

14 D. Schumann, A.M. Guinard-Baticle, P. Vanel and A. Talib, J. Electrochem. Soc., 134 (1987) 1128.

15 S. Partyka, M. Arnaud, M. Lindheimer and J.L. Cecile, Colloids Surf., 26 (1987) 141.

16 J. Gomez Becerra, R.C. Salvarezza and A.J. Arvia, J. Appl. Electrochem., 17 (1987) 779.

17 A. Bewick and J. Robinson, J. Electroanal. Chem., 60 (1975) 163; 71 (1976) 131.

18 A.M. Gaudin and G.S. Preller, AIME Min. Tech., 10 (1946) 2002.

19 C. Nguyen Van Huong, R. Parsons, P. Marcus, S. Montes and J. Oudar, J. Electroanal. Chem., 119 (1981) 137.

20 R.O. Lezna, N.R. de Tacconi and A.J. Arvia, in preparation.

21 J. Lipkowski, C. Nguyen Van Huong. C. Hinnen. R. Parsons and J. Chevalet, J. Electroanal. Chem., 143 (1983) 375. 
22 B.B. Damaskin, O.A. Petrii and V.V. Batrakov, Adsorption of Organic Compounds on Electrodes, Plenum Press, New York, 1971.

23 M. Kostelitz, J.L. Domange and J. Oudar, Surf. Sci., 34 (1973) 431.

24 C. Hinnen, C. Nguyen Van Huong, A. Rousseau and J.P. Dalbera, J. Electroanal. Chem., 95 (1979) 131.

25 R.O. Lezna, N.R. de Tacconi, C. Perdriel and A.J. Arvia, in S. Srinivasan, S. Wagner and H. Wroblowa (Eds.), Proc. Symp. on Electrode Materials and Processes for Energy Conversion and Storage, Philadelphia, 1987, The Electrochemical Society.

26 R. Adzic, E. Yeager and B.D. Cahan, J. Electrochem. Soc., 121 (1984) 474. 